\title{
CONTROL PREDICTIVO CENTRALIZADO Y DISTRIBUIDO DE UNA RED DE RECOGIDA DE AGUAS RESIDUALES
}

\author{
Antonio Cembellín Sánchez \\ Universidad de Salamanca, ETSII de Béjar, 37700 Béjar (Salamanca), España, cembe@usal.es \\ Mario Francisco Sutil, Pastora I. Vega Cruz \\ Universidad de Salamanca, Facultad de Ciencias,37008 Salamanca, España, mfs@usal.es, pvega@usal.es
}

\begin{abstract}
Resumen
En este trabajo se han desarrollado algoritmos de Control Predictivo aplicados a un sistema consistente en una red de recogida de aguas residuales en simulación, en particular, de tipo Centralizado y también controladores predictivos Distribuidos. Para obtención de estos últimos, se han empleado técnicas de sectorización, basadas en el análisis estructural, que proporcionan un criterio para descomponer un sistema complejo en subsistemas interconectados que se pueden controlar localmente junto con módulos de coordinación para conseguir procesos de negociación adecuados entre los controladores locales. Además, con el fin de mejorar el desempeño del sistema, se ha incorporado un método de generación dinámica de consignas. La comparación de los resultados obtenidos con ambas metodologías (centralizada y distribuida) permite extraer algunas conclusiones al final.
\end{abstract}

Palabras clave: Control Predictivo Distribuido, Control Predictivo Cooperativo, Sectorización de Sistemas de Gran Escala.

\section{INTRODUCCIÓN}

Actualmente, es evidente que las estaciones de tratamiento de aguas residuales urbanas (WWTPs) están altamente interconectadas con otros elementos, como redes de captación, sistemas de conducción, depósitos de almacenamiento, dentro del sistema urbano de aguas residuales (UWS), de tal modo que la evaluación de las estrategias de control de WWTPs deberían tratarse desde una perspectiva más amplia [1], [12]. Por esta razón, es necesario desarrollar herramientas integradas basadas en modelos para control y evaluación de UWS [4]. Otro aspecto importante, ha sido la reducción de la complejidad del modelo para poder realizar simulaciones a largo plazo [7] que permitan una mejor evaluación de los modelos y sistemas de control propuestos. En relación a estos últimos, se han propuesto controladores de tipo heurístico utilizando lógica borrosa [2] o sistemas jerárquicos en los que los set- points de reguladores que se sitúan a pie de proceso, se obtienen mediante la resolución de problemas de optimización lineal o no lineal [11].

Este documento presenta dos soluciones al problema de control planteado que posibilitan mantener una operación óptima teniendo en cuenta los requerimientos del sistema. Ambas se basan en la utilización de algoritmos de Control Predictivo basado en Modelos (MPC), de tipo Centralizado y Distribuido Cooperativo [6], este último más adecuado para sistemas de gran escala.

El Control Predictivo (Model Predictive Control o MPC, [9]) es una metodología de control que utiliza un modelo de predicción del proceso para calcular las variables manipuladas sobre un horizonte futuro con el fin de optimizar una determinada función de coste. En sistemas de gran escala, puede ser recomendable dividir el proceso global en subsistemas más simples para facilitar la aplicación de algoritmos de control predictivo [5]. En este caso, se utilizan modelos de predicción y funciones de coste locales, obteniéndose soluciones locales del problema de control global, llegando a lo que se denomina Control Predictivo Descentralizado.

Se habla de Control Predictivo Distribuido (DMPC) cuando se emplea un sistema de Control Predictivo Descentralizado en el que los controladores locales intercambian información entre ellos para calcular cada uno su propia solución local. Si ahora se considera que cada controlador local proporciona una solución para él y sus vecinos, de modo que un coordinador, aplicando un determinado criterio, decide cuál es la mejor solución para el sistema global de entre todas las propuestas [10], se trata de un sistema de Control Predictivo Distribuido Cooperativo (o Coordinado) [8]. Esta configuración requiere que exista comunicación entre los controladores locales y el coordinador, de modo que el sistema global opere en tiempo real. La implementación física del sistema de control con estos requerimientos se ajusta en gran medida al funcionamiento de los Sistemas Multiagente, 
constituidos por agentes como entidades inteligentes que se pueden comunicar para lograr un objetivo.

Este artículo presenta, en primer lugar, el modelo matemático del sistema. En el punto siguiente, se detalla la sectorización del mismo. Continúa con la descripción de los algoritmos de control MPC centralizado y distribuido, mostrando los resultados correspondientes en cada caso, para finalizar con las conclusiones del trabajo.

\section{MODELO MATEMÁTICO DE UNA RED DE RECOGIDA DE AGUAS RESIDUALES}

\subsection{DESCRIPCIÓN DEL SISTEMA}

El sistema de recogida de aguas residuales que se utiliza como banco de pruebas (benchmark) [13] para ensayar algoritmos de control (figura 1) está formado por 6 zonas de recogida de agua residual y de lluvia (numeradas del 1 al 6 en la figura), 6 depósitos de almacenamiento de aguas residuales (ST1,..,ST6, uno de ellos, ST5, se encuentra off-line), conducciones de agua residual, 5 válvulas y una bomba para control de caudal, y una estación depuradora de aguas residuales (WWTP). Se trata de recoger toda el agua residual y conducirla hasta la planta de tratamiento manteniendo un caudal de aporte con la menor variabilidad posible y lo más próximo a su valor nominal. Ello se consigue reteniendo el volumen recogido en los depósitos, en el caso de que sea relativamente grande, o liberando ese volumen cuando el caudal recogido sea pequeño.

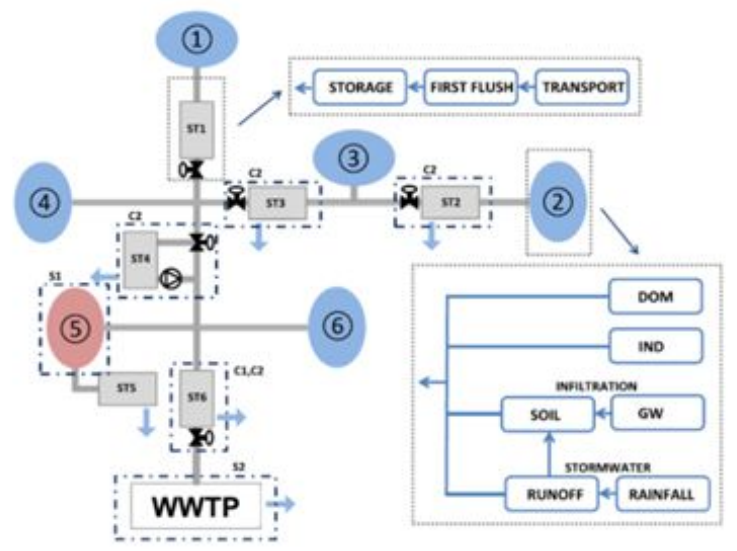

Figura 1: Red de recogida de aguas residuales

\subsection{MODELO SISTEMA \\ SIMPLIFICADO \\ DEL}

Con el fin de agilizar el proceso de diseño y validación de los algoritmos de control, se ha desarrollado un modelo simplificado del proceso. Las simplificaciones consisten en considerar únicamente un modelo hidráulico, prescindiendo de variables que aparecen en el benchmark representando concentraciones de contaminantes, no contemplar la posibilidad de desborde en los depósitos ST2, ST3, ST4 y ST6, puesto que ello implicaría la introducción de no linealidades en el sistema, y la eliminación del depósito ST5, ya que no es posible ejercer control sobre él. Las conducciones entre depósitos y con la WWTP se modelan como sistemas de $1^{\text {er }}$ orden con una dinámica muy lenta, de acuerdo con su longitud. El modelo matemático simplificado del proceso es el siguiente [11]:

$$
\begin{aligned}
& x_{1}(k+1)=x_{1}(k)+\frac{T}{A_{1}}\left[d_{1}(k)-u_{1}(k)\right] \\
& x_{2}(k+1)=x_{2}(k)+\frac{T}{A_{2}}\left[d_{2}(k)-u_{2}(k)\right] \\
& x_{3}(k+1)=x_{3}(k)+\frac{T}{A_{3}}\left[x_{6}(k)-u_{3}(k)\right] \\
& x_{4}(k+1)=x_{4}(k)+\frac{T}{A_{4}}\left[d_{3}(k)+u_{1}(k)+u_{3}(k)-u_{4}(k)\right] \\
& x_{5}(k+1)=x_{5}(k)+\frac{T}{A_{5}}\left[x_{8}(k)-u_{5}(k)\right] \\
& x_{6}(k+1)=\left(1-\frac{T}{\tau_{3}}\right) x_{6}(k)+\left(\frac{T}{\tau_{3}}\right)\left[d_{6}(k)+u_{2}(k)\right] \\
& x_{8}(k+1)=\left(1-\frac{T}{\tau_{8}}\right) x_{8}(k)+\left(\frac{T}{\tau_{8}}\right)\left[d_{4}(k)+d_{5}(k)+x_{7}(k)\right] \\
& x_{7}(k+1)=\left(1-\frac{T}{\tau_{7}}\right) x_{7}(k)+\left(\frac{T}{\tau_{7}}\right) u_{4}(k) \\
& x_{9}(k)+\left(\frac{T}{\tau_{9}}\right) u_{5}(k) \\
& \left.x_{9}\right) \\
& \left.x_{9}\right) \\
& \left.x_{9}\right)
\end{aligned}
$$

A partir de estas ecuaciones, se obtiene el modelo linealizado del sistema que se va a usar como modelo de predicción en el algoritmo MPC:

$$
\mathbf{x}(k+1)=\mathbf{A x}(k)+\mathbf{B}_{p} \mathbf{u}(k)+\mathbf{B}_{d} \mathbf{d}(k)
$$

$$
\mathbf{x}(k+1)=\mathbf{A x}(k)+\mathbf{B}\left[\begin{array}{c}
\mathbf{u}(k) \\
\mathbf{d}(k)
\end{array}\right], \mathbf{B}=\left[\begin{array}{ll}
\mathbf{B}_{p} & \mathbf{B}_{d}
\end{array}\right]
$$


Los estados considerados son los niveles de los depósitos ST1, ST2, ST3, ST4 y ST6 $\left(x_{1}, \ldots, x_{5}\right)$ y los caudales de salida de los elementos de enlace que comunican los depósitos ST2 y ST3, ST4 y ST6 (entre estos hay dos elementos de enlace en serie) y el depósito ST6 y la WWTP, que se corresponden con los estados $\left(x_{6}, \ldots, x_{9}\right)$. Los elementos de enlace son conducciones que conectan los caudales recogidos en cada zona con los depósitos y los depósitos entre sí y con la estación depuradora. Se han incorporado de forma que el modelo simplificado es análogo al benchmark. Los caudales de salida de los elementos de enlace de las zonas de recogida de agua $1,2,4,5$ y 6 , y el caudal recogido en la zona 3 , se considerarán como perturbaciones medibles sobre el proceso: $\left(d_{1}, \ldots d_{6}\right)$. El resto de parámetros del modelo, se definen en la tabla 1.

Las entradas del sistema son los caudales deseados a la salida de cada uno de los depósitos (variables manipuladas): $\left(u_{1}, \ldots, u_{5}\right)$.

Se consideran todos los estados como salidas del sistema, con especial interés en el caudal de entrada a la depuradora.

Tabla 1: Parámetros del sistema.

\begin{tabular}{|c|c|c|}
\hline Parámetro & Uds. & Valores \\
\hline $\begin{array}{l}\mathrm{A}_{1}, \ldots, \mathrm{A}_{5} \text { áreas de los } \\
\text { depósitos }\end{array}$ & $m^{2}$ & $\begin{array}{l}1188,252,348, \\
852,852\end{array}$ \\
\hline $\begin{array}{l}\mathrm{c} 01, \ldots, \mathrm{c} 05 \\
\text { coeficientes } \\
\text { descarga }\end{array}$ & $m^{5 / 2} / d$ & $\begin{array}{l}1.89,0.40,0.55, \\
1.36,6.12\left(\times 10^{4}\right)\end{array}$ \\
\hline $\begin{array}{l}\mathrm{h}_{\max 1}, \ldots, \mathrm{h}_{\max 5} \text { alturas } \\
\text { de los depósitos }\end{array}$ & $m$ & 5 (para todos) \\
\hline $\begin{array}{l}\mathrm{h}_{\min 1}, \ldots, \mathrm{h}_{\min 5} \text { niveles } \\
\text { mínimos }\end{array}$ & $m$ & 0 (para todos) \\
\hline $\begin{array}{l}\mathrm{q}_{\max 1}, \ldots, \mathrm{q}_{\max 9} \text { caudales } \\
\text { máximos a la salida de } \\
\text { los elementos de } \\
\text { enlace }\end{array}$ & $m^{3} / d$ & $\begin{array}{l}5.99, \quad 1.27, \quad 3.02, \\
4.29,4.29,15.06, \\
4.29, \quad 23.64, \quad 6 \\
\left(\times 10^{4}\right)\end{array}$ \\
\hline T período de muestreo & $d$ & 0.0021 \\
\hline $\begin{array}{l}\tau_{1}, \ldots, \tau_{9} \text { constantes de } \\
\text { tiempo de los } \\
\text { elementos de enlace }\end{array}$ & $d$ & $\begin{array}{ll}0.0313, & 0.0104, \\
0.0104, & 0.0208, \\
0.0208, & 0.073, \\
0.0208, & 0.0104, \\
0.0104 & \end{array}$ \\
\hline $\begin{array}{l}\mathrm{u}_{\max 1}, \ldots, \mathrm{u}_{\max 5} \text { caudales } \\
\text { máximos a la salida de } \\
\text { los depósitos }\end{array}$ & $m^{3} / d$ & $\begin{array}{l}5.98, \quad 1.27, \quad 1.75, \\
4.29,19.34\left(\times 10^{4}\right)\end{array}$ \\
\hline
\end{tabular}

obtenido su grafo directo [14] (figura 2) el cual permitirá conocer las interacciones entre las distintas variables que intervienen en el proceso y determinar el mejor modo de dividir el sistema global en subsistemas que tengan un mínimo grado de acoplamiento [3].

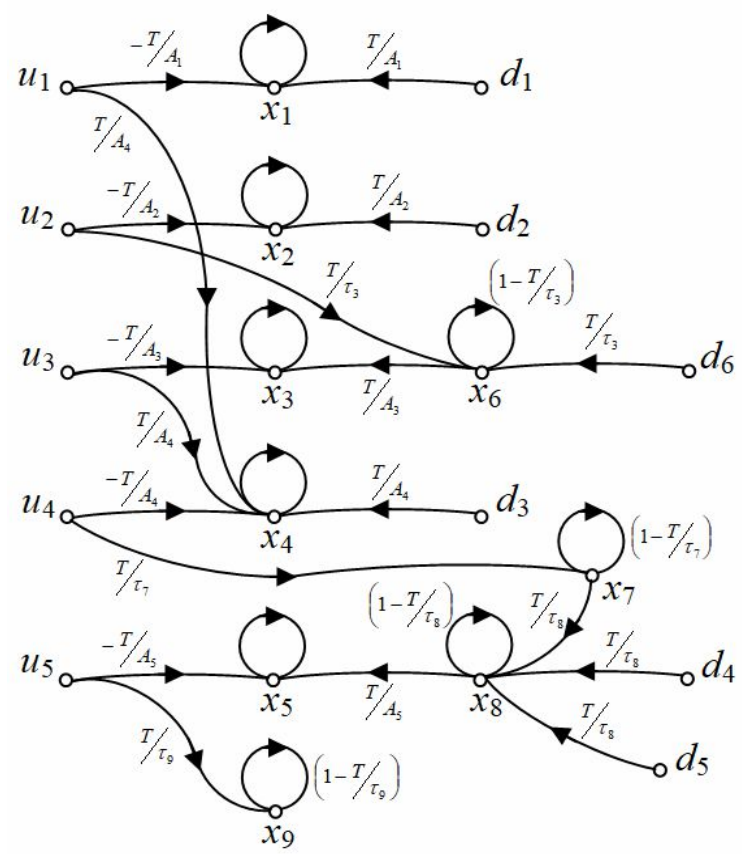

Figura 2: Grafo directo del sistema objeto de estudio

El grafo muestra un sistema acoplado por las entradas, lo cual facilita el proceso de sectorización. Por simplicidad, en principio se van a considerar únicamente dos subsistemas acoplados por la entrada $u_{4}$.

Sistema1

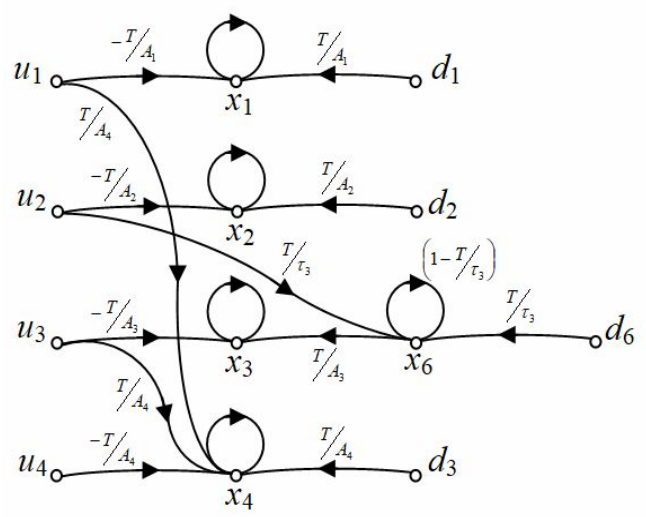

\section{SECTORIZACIÓN DEL SISTEMA}

A partir del modelo matemático del sistema y mediante el análisis estructural del mismo, se ha 
Sistema 2

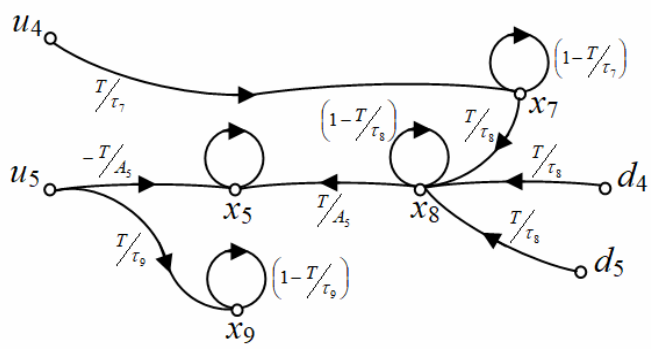

Figura 3: Sectorización del sistema

Los modelos matemáticos locales de cada subsistema que serán utilizados por los algoritmos de Control Predictivo Distribuido son:

$$
\begin{aligned}
& \mathbf{x}_{1}(k+1)=\mathbf{A}_{1} \mathbf{x}_{1}(k)+\mathbf{B}_{1}\left[\begin{array}{l}
\mathbf{u}_{1}(k) \\
\mathbf{d}_{1}(k)
\end{array}\right], \text { donde: } \\
& \mathbf{x}_{1}=\left(x_{1}, \ldots, x_{4}, x_{6}\right), \mathbf{u}_{1}=\left(u_{1}, \ldots, u_{4}\right), \\
& \mathbf{d}_{1}=\left(d_{1}, \ldots, d_{4}, d_{6}\right) \\
& \mathbf{x}_{2}(k+1)=\mathbf{A}_{2} \mathbf{x}_{2}(k)+\mathbf{B}_{2}\left[\begin{array}{l}
\mathbf{u}_{2}(k) \\
\mathbf{d}_{2}(k)
\end{array}\right], \text { donde: } \\
& \mathbf{x}_{2}=\left(x_{5}, x_{7}, x_{8}, x_{9}\right), \mathbf{u}_{2}=\left(u_{4}, u_{5}\right), \\
& \mathbf{d}_{2}=\left(d_{4}, d_{5}\right)
\end{aligned}
$$

Las matrices $\mathbf{A}_{1}, \quad \mathbf{B}_{1}, \quad \mathbf{A}_{2} \quad$ y $\quad \mathbf{B}_{2}$ se forman seleccionando adecuadamente las filas y columnas de A y $\mathbf{B}$ de la expresión (2).

\section{CONTROL CENTRALIZADO}

\section{PREDICTIVO}

\subsection{DESCRIPCIÓN DEL ALGORITMO}

El objetivo de control es asegurar un caudal en la depuradora que aproveche al máximo su capacidad sin superar un valor máximo, evitando en lo posible desbordamientos en los depósitos y en la propia estación, y minimizando también los costes de operación.

El algoritmo empleado para el Control Predictivo Centralizado utiliza un modelo lineal del proceso en el espacio de estados para predicción que incluye las perturbaciones y una función de coste que calcula los caudales de salida de los depósitos para una operación óptima.
La función de coste o función objetivo del MPC es una forma cuadrática que tiene en cuenta tanto los errores de seguimiento en los estados, como los incrementos en la secuencia de control respecto la referencia de caudal (penalizando los esfuerzos de control), suponiendo que los horizontes de predicción y control coinciden y su valor es $N$ :

$$
J(k)=\sum_{i=1}^{N-1}\left[\left\|\hat{\mathbf{x}}(k+i)-\mathbf{x}_{r e f}\right\|_{Q(k)}^{2}+\left\|\mathbf{u}(k+i)-\mathbf{u}_{r e f}\right\|_{R}^{2}\right]+\mathbf{x}^{T}(k+N) P \mathbf{x}(k+N)
$$

donde:

$$
\begin{aligned}
& \mathbf{x}_{r e f}=\left(x_{1 r e f}, x_{2 r e f}, \ldots, x_{9 r e f}\right) \\
& \mathbf{u}_{r e f}=\left(u_{1 r e f}, u_{2 r e f}, \ldots, u_{5 r e f}, d_{1}, d_{2}, \ldots, d_{6}\right)
\end{aligned}
$$

El problema de optimización que resuelve el MPC centralizado sería:

$$
\mathbf{u}_{k}^{*}=\underset{\mathbf{u}(k+i)}{\arg \min } J(k)
$$

sujeto a:

$$
\begin{aligned}
& \hat{\mathbf{x}}(i+1)=\mathbf{A} \hat{\mathbf{x}}(i)+\mathbf{B u}(i) \\
& \hat{\mathbf{x}}(0)=\mathbf{x}(k) \\
& 0 \leq \hat{x}_{1}(k+i) \leq h_{\max 1}, i=1, \ldots, N \\
& 0 \leq \hat{x}_{j}(k+i) \leq q_{\max }, i=1, \ldots, N, j=6, \ldots, 9 \\
& 0 \leq u_{j}(k+i) \leq u_{\max j}, i=1, \ldots, N, j=1, \ldots, 5 \\
& u_{j}(k+i)=d_{l}, i=1, \ldots, N, j=6, \ldots, 11, l=1, \ldots, 6
\end{aligned}
$$

Se ha considerado una matriz $Q$ variable, de modo que, si se produce desborde en algún depósito, el peso correspondiente se modifica con el fin de evitarlo en lo posible, excepto para el ST1, ya que no se permiten desbordes en él mediante la inclusión de una restricción en la formulación del MPC. Los elementos no nulos de la diagonal de $Q$ son:

$$
q_{i i}=\left\{\begin{array}{cc}
f_{i} & x_{i} \leq h_{\max i} \\
f_{i}\left(1+10\left(x_{i}-h_{\max i}\right)^{2}\right) & x_{i}>h_{\max i}
\end{array}\right.
$$

donde $i=2, \ldots, 5$ y $f_{i}$ es un parámetro de sintonía del MPC.

El resto de los elementos de la diagonal de $Q$ son iguales a cero ya que se refieren a los caudales de salida de los elementos de enlace $\left(x_{6}, \ldots, x_{9}\right)$.

Para conseguir una operación óptima del sistema, se ha incluido un controlador jerárquico que en una capa superior modifica las consignas de nivel según la estrategia de distribuir el volumen de agua actual 
entre todos los depósitos de la forma más uniforme posible teniendo en cuenta su capacidad. Ello se consigue calculando para cada uno su nivel de referencia en función de la capacidad total de la red y la capacidad de ese depósito [11]:

$$
x_{i r e f}(k)=\left(\frac{V_{G}(k)}{A_{i}}\right) v_{i}, v_{i}=\frac{V_{i}}{\sum_{j=1}^{5} V_{j}}
$$

donde $x_{\text {iref }}(k)$ es el nivel de referencia para el depósito $i$ en el instante $k ; V_{G}(k)$ es el volumen total ocupado en el instante $k ; v_{i}$ es un factor que representa el peso de la capacidad del depósito $i$ en el volumen total disponible (suma de todas las capacidades de los depósitos); $V_{i}$ y $A_{i}$ son la capacidad máxima y la superficie del depósito $i$, respectivamente.

Las referencias de caudal que se corresponden con las de los niveles, se calculan teniendo en cuenta el nivel deseado para cada depósito según la expresión (8):

$$
u_{\text {iref }}(k)=c_{0 i} \sqrt{x_{\text {iref }}(k)}, i=1, \ldots, 5
$$

\subsection{RESULTADOS}

En este apartado se muestran los resultados obtenidos sobre el modelo simplificado contemplando diversos escenarios utilizando el MPC centralizado descrito.

Los resultados que se muestran, se han obtenido seleccionando como pesos en la función de coste: $f_{i}=1, i=1,2,3 ; f_{4}=10 ; f_{5}=50 ; f_{9}=10^{-6}$; $f_{j}=0, j=6,7,8$.

Esta selección de pesos prioriza mantener el caudal en la depuradora lo más próximo al valor deseado y los niveles de los depósitos ST6 y ST4 los más cerca posible a sus referencias, por ser los depósitos que se encuentran más próximos a la WWTP.

Para la penalización del esfuerzo de control se han fijado unos valores $r_{i i}=1, i=1, \ldots, 5$, que son los valores de la diagonal de $R$. En la figura 3 se muestran los resultados obtenidos en condiciones nominales de operación para un caudal deseado de entrada a la depuradora de $50.000 \mathrm{~m}^{3} / d$. en un intervalo de 3 días. Inicialmente, se ha supuesto que los niveles de los depósitos son 1, 1, 2, 2.5 y $1.5 \mathrm{~m}$ y que los caudales en los elementos de enlace son todos iguales a $1.500 \mathrm{~m}^{3} / d$. Para los caudales recogidos las zonas $1, \ldots, 6$ (perturbaciones del sistema) se han tomado los valores de 10.000, 6.000, 6.000, 6.000, 20.000 y $2.000 \mathrm{~m}^{3} / d$, respectivamente, que se mantienen constantes en el tiempo de simulación (caso 1).
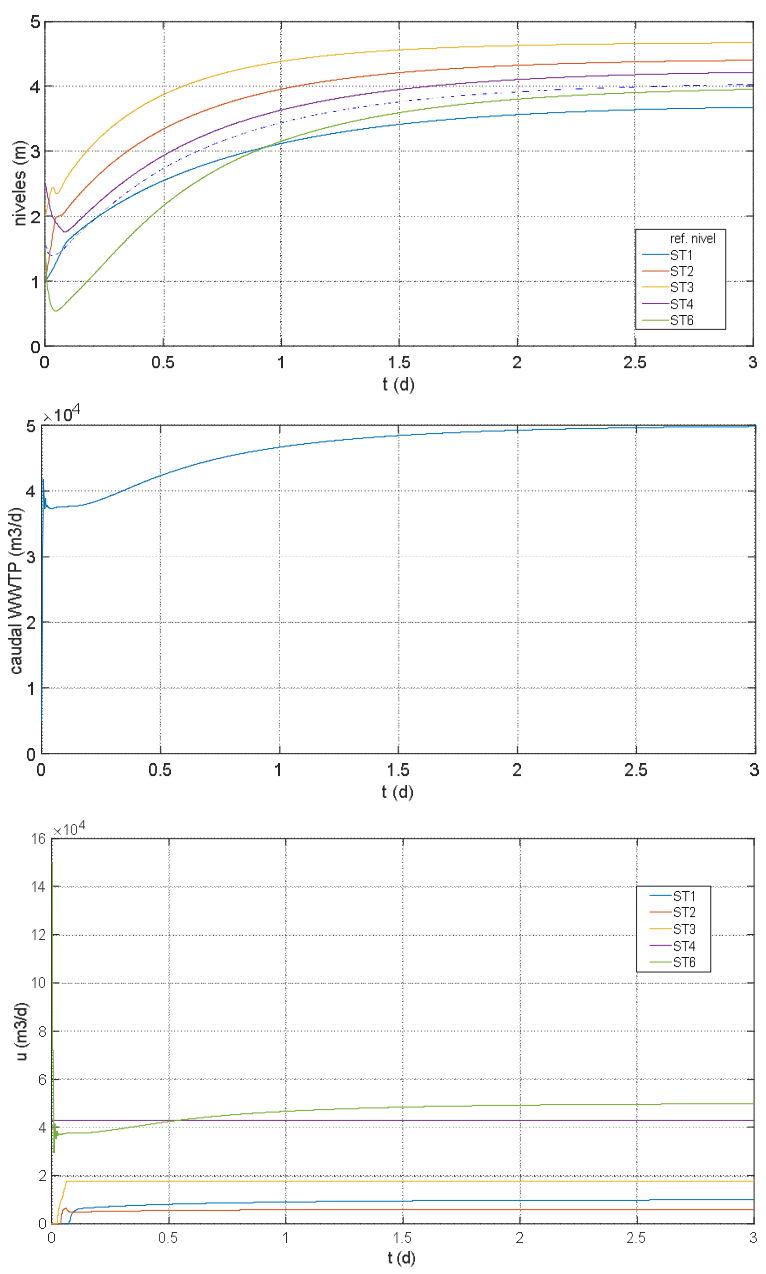

Figura 4: Resultados con MPC Centralizado (caso 1)

Se puede observar que las consignas de nivel generadas son iguales para todos los depósitos porque todos tienen la misma de altura. Éstas van creciendo debido a que, para las perturbaciones existentes, es necesario almacenar más agua de forma que no se supere el caudal de entrada a la depuradora. En las pruebas realizadas, las consignas se modifican cada dos períodos de muestreo.

Con este mismo controlador, se ha considerado un segundo caso en el que los caudales recogidos en cada zona varían como se muestra en la figura 5 .

De los resultados obtenidos (figura 6), se deduce que en este caso es más difícil que el sistema alcance los niveles deseados y lo mismo ocurre con el caudal de entrada a la WWTP. Ello es debido a la gran variabilidad de los caudales recogidos, los cuales constituyen fuertes perturbaciones al sistema. 


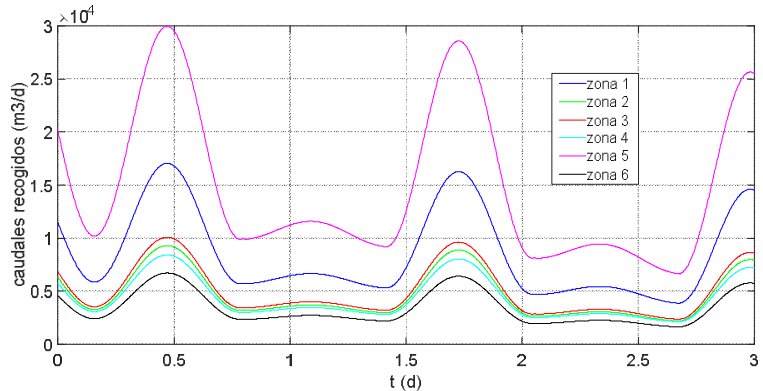

Figura 5: Caudales recogidos en cada zona (caso 2)
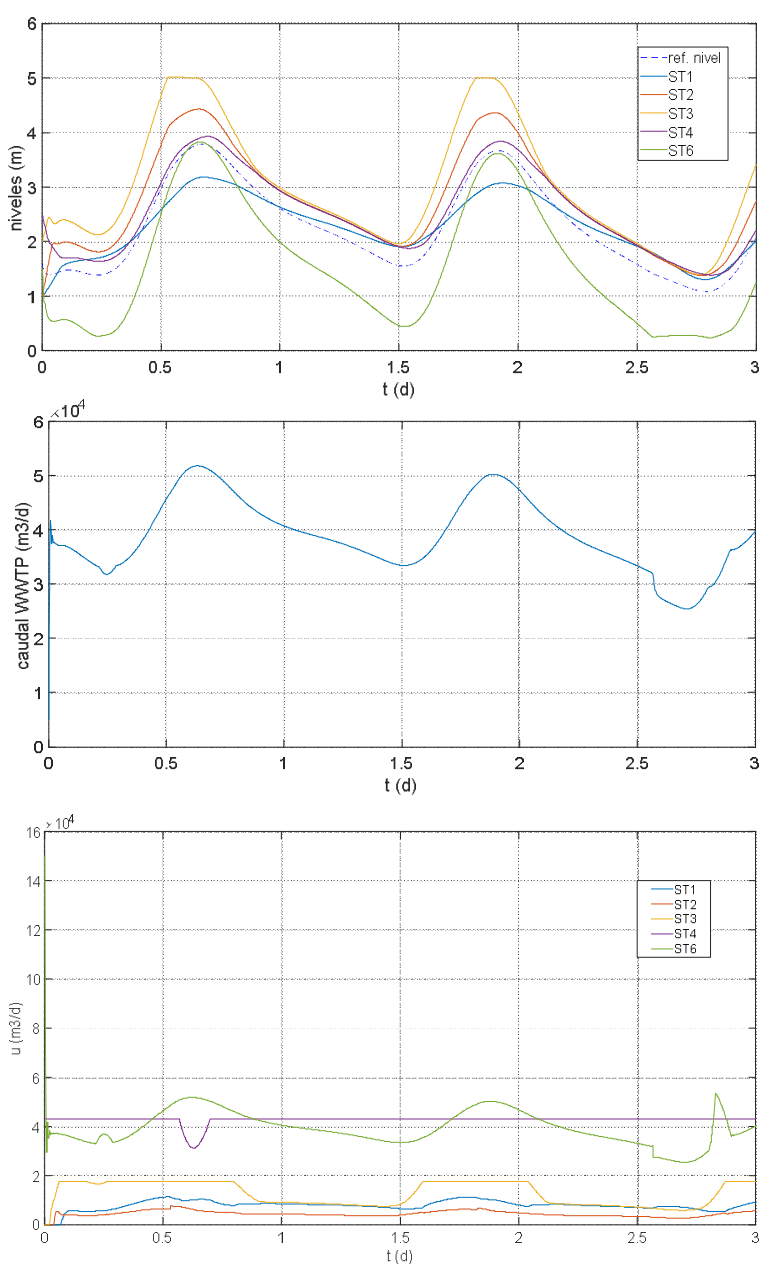

Figura 6: Resultados con MPC Centralizado (caso 2)

\section{CONTROL DISTRIBUIDO}

\subsection{DESCRIPCIÓN DEL ALGORITMO}

El algoritmo consiste en la resolución cada dos períodos de muestreo de dos problemas de optimización locales para cada uno de los subsistemas 1 y 2 y el posterior intercambio de información entre ambos. Para ello, se consideran modelos de predicción locales (3), funciones de coste locales y restricciones locales que se obtienen a partir del sistema global teniendo en cuenta la sectorización del mismo. Los pasos que constituyen este algoritmo distribuido, se describen a continuación con ayuda de la figura 7:

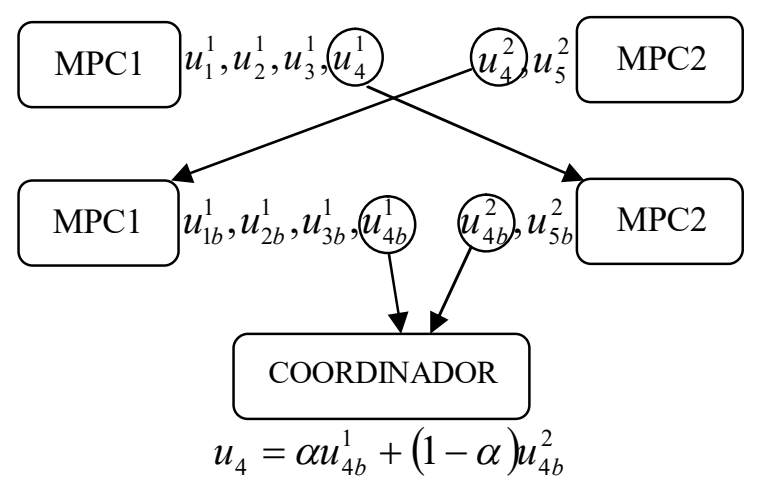

Figura 7: Algoritmo de DMPC

PASO 1: Se resuelven dos problemas locales de control predictivo (MPC1 y MPC2), obteniéndose las soluciones locales $u_{1}^{1}, u_{2}^{1}, u_{3}^{1}, u_{4}^{1} \mathrm{y} u_{4}^{2}, u_{5}^{2}$ para cada subsistema.

PASO 2: Los controladores locales intercambian las soluciones obtenidas para la variable compartida $u_{4}$ y vuelven a resolver los problemas locales incorporando como restricciones de igualdad en el cálculo, los valores recibidos de su vecino.

PASO 3: Se obtienen nuevas soluciones locales $u_{1 b}^{1}, u_{2 b}^{1}, u_{3 b}^{1}, u_{4 b}^{1}$ y $u_{4 b}^{2}, u_{5 b}^{2}$ por parte de cada controlador, donde debido a las restricciones de igualdad añadidas: $u_{4 b}^{1}=u_{4}^{2}$ у $u_{4 b}^{2}=u_{4}^{1}$.

PASO 4: Finalmente, se toman las dos soluciones propuestas por cada controlador para $u_{4}$ y se aplica al sistema el valor medio: $u_{4}=\alpha u_{4 b}^{1}+(1-\alpha) u_{4 b}^{2}$, $\alpha=0.5$. Para el resto de entradas, se aplican los últimos valores calculados por cada controlador local.

\subsection{RESULTADOS EN SIMULACIÓN CON MPC DISTRIBUIDO}

La figura 8 muestra los resultados de simulación del sistema donde se ha aplicado el algoritmo anterior con las mismas condiciones que se fijaron para el control centralizado (caso 1), adaptadas a la situación actual. Se puede apreciar un comportamiento muy parecido al del MPC centralizado, dado que no hay un alto grado de acoplamiento entre los dos 
subsistemas considerados y los caudales recogidos son constantes.
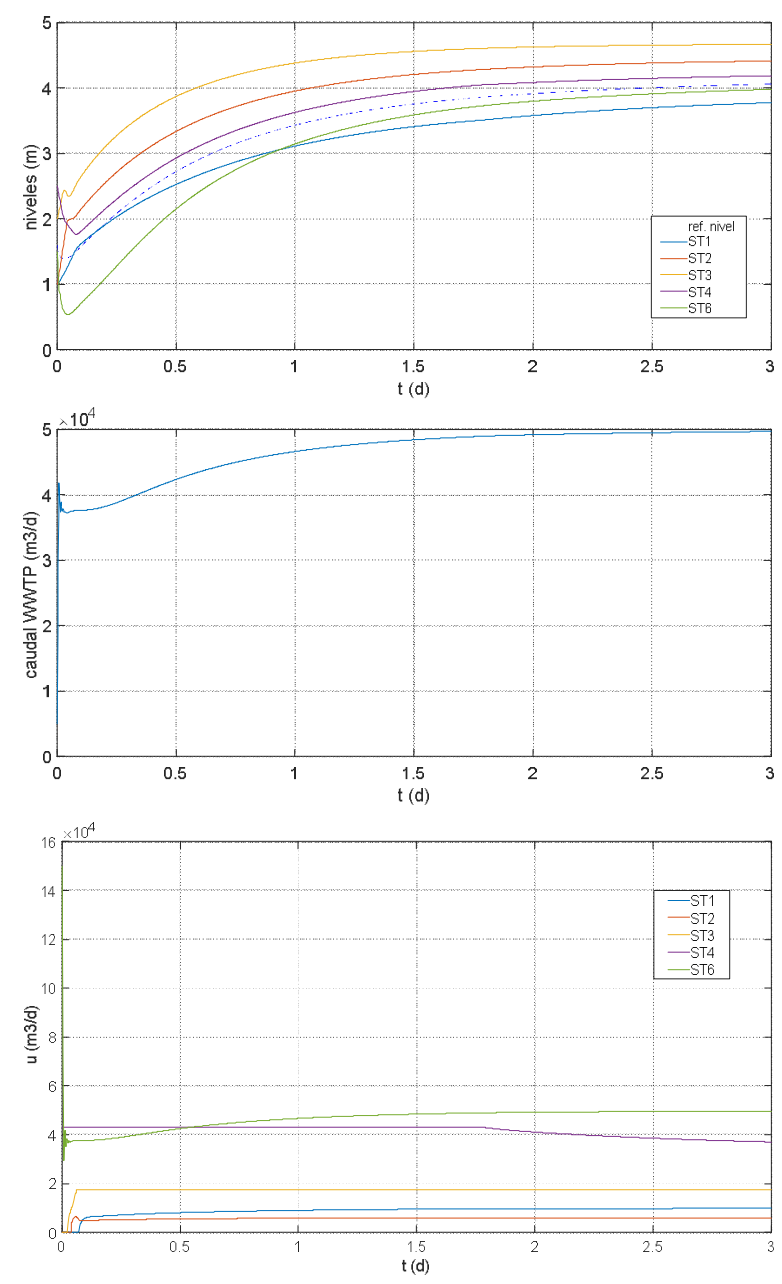

Figura 8: Resultados con MPC distribuido (caso 1)

En el segundo caso, los resultados (figura 9) son diferentes a los obtenidos con el MPC centralizado debido a la gran variabilidad de los caudales recogidos que provoca que el desempeño sea peor, siendo necesaria una resintonía de los controladores locales.

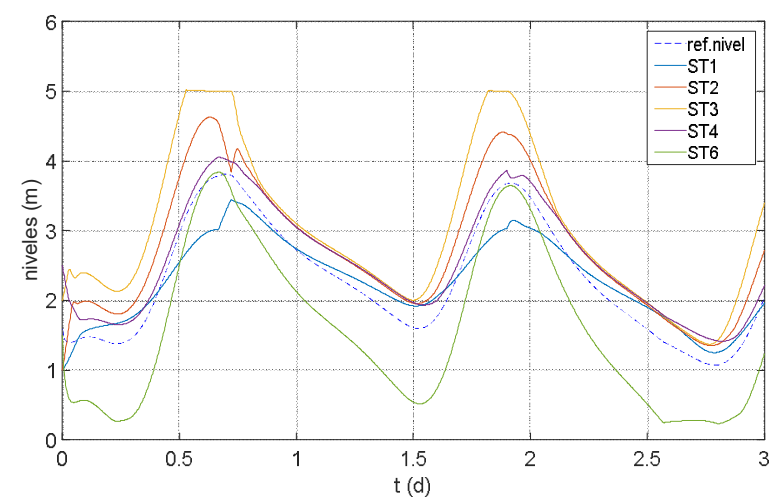

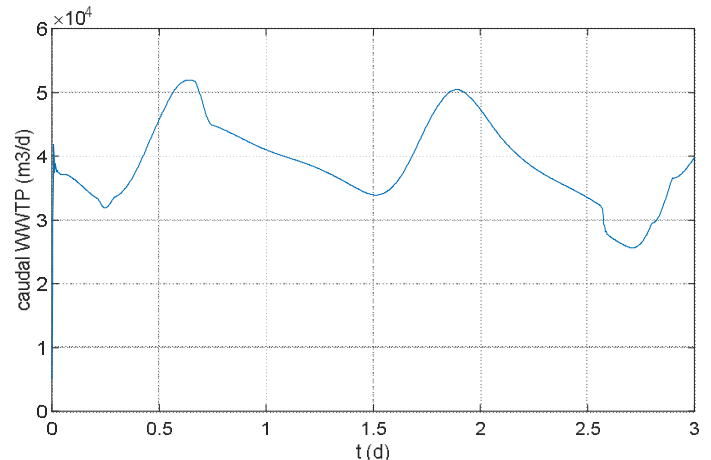

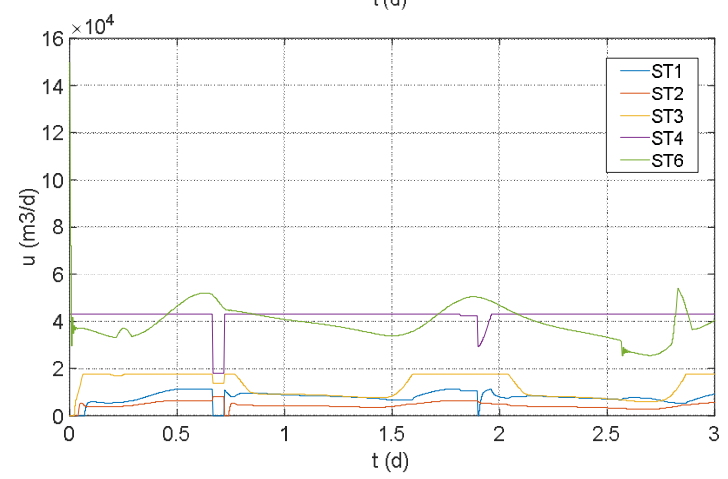

Figura 9: Resultados con MPC distribuido (caso 2)

\section{CONCLUSIONES}

En este trabajo se ha sintetizado un controlador MPC centralizado y otro distribuido aplicado a un sistema de recogida de aguas residuales con buenos resultados. En esta línea, se propone continuar desarrollando algoritmos de Control Distribuido Cooperativo más complejos para este mismo sistema o para otros de mayor escala, así como la consideración de situaciones de funcionamiento más realistas.

\section{Agradecimientos}

Los autores agradecen el apoyo del Gobierno Español a través del proyecto MINECO DPI201567341-C2-1-R.

\section{English summary}

CENTRALIZED AND DISTRIBUTED MODEL-BASED PREDICTIVE CONTROL APPLIED TO A SEWER NETWORK

\author{
Abstract \\ In this work, Model-Based Predictive Control \\ algorithms applied to a simulated sewer network.
}


have been developed, particularly, Centralized and Distributed Predictive Control. For the distributed algorithms, sectorization techniques based on structural analysis have been applied providing a criterion to decompose a complex system into interconnected subsystems that may be controlled in a local way together with coordination modules to accomplish proper negotiation processes between local controllers. Moreover, a dynamic set-point generation method has been proposed to improve the system performance. A comparison of results obtained with both methodologies (centralised and distributed approach) allows some conclusion to be drawn and given at the end.

Keywords: Distributed Model-Based Predictive Control, Cooperative Predictive Control, Complex Systems Sectorization.

\section{Referencias}

[1] Bach, P. M., Rauch, W., Mikkelsen, P.S., McCarthy, D. T., Deletic, A., (2014) A critical review of integrated urban water modelling urban drainage and beyond. Env. Mod. Soft. 54, 88-107.

[2] Barbu, M., Vilanova, R., (2016) Fuzzy Control Applied on a Benchmark Simulation Model for Sewer Networks. 20th International Conference on System Theory, Control and Computing (ICSTCC), October 13-15, Sinaia, Romania.

[3] Barcelli, D., (2008) Optimal decomposition of Barcelona's water distribution network system for applying distributed Model Predictive Control. Master thesis. Universitat Politècnica de Cataluña-IRI-Universitá degli Study di Siena.

[4] Benedetti, L., Langeveld, J. G., Corneau, A., Corominas, L., Daigger, G. T., Martin, C., Mikkelsen, P. S., Vezzaro, L., Weijers, S., Vanrolleghem, P. A., (2013) Modelling and monitoring of integrated urban wastewater systems: review on status and perspectives. Wat. Sci. Tech. 68 (6), 1203-1215.

[5] Farina, M., Scattolini, R., (2018) Distributed MPC for Large-Scale Systems. Ed. Springer.
[6] Ferramosca, A., Limon, D., Alvarado, I., Camacho, E. F., (2013) Cooperative distributed MPC for tracking. Automatica, 49(4), 906-914.

[7] Fu, G., Khu, S.-T., Butler, D., (2009a) Use of surrogate modelling for multiobjective optimisation of urban wastewater systems. Wat. Sci. Tech. 60 (6), 1641-1647.

[8] Li, Z., Duan, Z., (2015) Cooperative control of multi-agent systems. A consensus region approach. Ed. CRC Press.

[9] Maciejowski, J. M., (2002) Predictive Control with Constraints. Ed. Pearson Education.

[10] Maestre, J.M., Muñoz de la Peña, D., Camacho, E.F., Alamo, T., (2011) Distributed model predictive control based on agent negotiation. Journal of Process Control, 21, 5, pp. 685-697.

[11] Marinaki, M., (2005) Optimal Real-time Control of Sewer Networks. Ed. Springer.

[12] Rauch, W., Bertrand-Krajewski, J. L., Krebs, P., Mark, O., Schilling, W., Schütze, M., Vanrolleghem, P. A., (2002) Deterministic modelling of integrated urban drainage systems. Wat. Sci. Tech. 45 (3), 81-94.

[13] Saagi, R., Flores-Alsina, X., Fu, G., Butler, D., Gernaey, K.V., Jeppsson, U., (2016) Catchment \& sewer network simulation model to benchmark control strategies within urban wastewater systems. Ed. Elsevier.

[14] Siljak, D.D., (1991) Decentralized Control of Complex Systems. Ed. Dover.

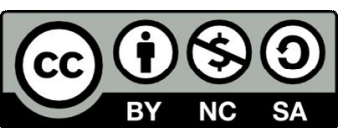
(C) 2019 by the authors. Submitted for possible open access publication under the terms and conditions of the Creative Commons Attribution CC BY-NC-SA 4.0 license (https://creativecommons.org/licenses/bync-sa/4.0/deed.es). 